\title{
Computation of Smoothed Local Symmetries on a MIMD Architecture
}

\author{
M. W. Wright \\ Engineering Department, University of Cambridge \\ Cambridge England
}

\begin{abstract}
An algorithm is presented to compute smoothed local symmetries on a MIMD architecture. Based on Brady and Scott's Connection Machine algorithm, it is adapted to reduce the synchronisation and communication overheads involved. Using an evidence accumulation approach similar to the Hough transform for circle detection, this algorithm can compute both local and rotational symmetries simultaneously. Implementation on an array of transputers is described and the results discussed.
\end{abstract}

\section{Introduction}

Shape Description is an important area in computer vision where many approaches exist [8]. The main value of shape descriptors lies in the fact that they can be used as intermediate representations within a complete object recognition system [6]. Of particular interest in this respect is the Smoothed Local Symmetries (SLS) descriptor $[4,1,3]$ which encodes both the boundary and the region of a shape. Here we focus on the region based portion.

In [5] Brady and Scott introduce a parallel algorithm to compute the SLS representation on the Connection Machine. In their algorithm each point in the image plane is associated with a processor. Processors on the boundary of a shape store the orientation of the boundary contour at that point and these contour processors simultaneously propagate messages along their inward normals. Other processors look for instances of messages passing "almost overhead almost instantaneously" [5]. In such an event the two message origins on the boundary are deemed to be locally symmetric and a message is sent to the corresponding SLS point, i.e. the bisector of the chord between them.

Figure 1 shows the justification for this approach. Points A and B are locally symmetric because the angles between their normals and the chord which joins them are equal. A and B are also vertices of an isoceles triangle with point $\mathrm{O}$, the intersection of their two normals. As the sides $\mathrm{AO}$ and $\mathrm{BO}$ of the triangle are equal, any messages sent simultaneously from $\mathrm{A}$ and $\mathrm{B}$ along their inward normals will reach $O$ together and thus signify that $A$ and $B$ are locally symmetric about the point $\mathrm{S}$, the bisector of the chord between them.

\section{The Adapted MIMD Algorithm}

The key to efficient computation on MIMD architectures lies in the judicious decomposition of the problem. In particular we should divide the problem such that communication and synchronisation overheads are minimised. 
Synchronisation requirements can be reduced if each pixel is considered a bin into which data is dropped by overflying messages. Two pieces of information are deposited, the distance the message has travelled and its origin. After all messages have propagated across the shape the bins are inspected for equal distances signifying a local symmetry. The advantage of this approach is no globally synchronised signal is required.

Data transfer reduction also depends on task decomposition. We could give each node a copy of the whole shape and a unique portion of the boundary from which to propagate messages as shown in figure 2 . This would require local copies of bins on each transputer, bin contents would have to be pooled using large data transfers.

A better way to split up the problem is to give each node a complete copy of the boundary, but only have them propagate within small and disjoint intervals along all the normals. For example, node one propagates from 0 to 5 pixels along all the normals, node two propagates from 5 to 10 pixels, etc. Note that nodes do not repeat processing performed by other nodes, they jump immediately to the lower bound of their interval by use of real arithmetic. There is no need for inter-node communication as all possible local symmetry points, ie with lengths of the same order, are contained on the same node.

Figure 2 shows this new decomposition leads to a reduction in the total computations performed. Messages from Transputers A and B cross at $\mathrm{O}$ and thus are potentially locally symmetric. However this is not the case as the messages have travelled greatly different lengths. Using boundary decomposition we still take time to consider and discard them. The new interval decomposition method only considers intersecting normals of similar lengths. This effect becomes more pronounced as processors are added to the array.

Rotational Symmetries can also be detected. Messages propagated from a circular arc reach the centre together swamping the finite capacity of the Connection Machine processors. This is not so on a MIMD architecture which can allocate voting space dynamically. Note however Brady uses Fleck's criteria for rotational symmetry [7] and can thus cope with "near circular" shapes.

\section{Implementation and Results}

The MIMD architecture used for the implementation of the algorithm was an array of twelve T800 Transputers connected to a host Sun workstation via a T414. Test images from the host were preprocessed on the T414 including Canny edge detection, edge linking and boundary tracking. The message propagation and "voting" was carried out on the main array before uploading to the host for display.

In figure 3 we can see the results of applying the algorithm to a square and a "T" shape, all the axes of symmetry are present. Figure 4 shows a comparison of the SLS and the Symmetric Axis Transform (SAT). The SLS does not distort axes when new features such as a notch or occluding object are added as the SAT does. This is an important property when the SLS is used for object recognition.

Figure 5 shows a poor SLS for a wrench where only the main axis is picked out. Problems include noise giving errors in normal angle and position, normals of similar origin and angle give multiple SLS points and normals can cross but 
not vote in same bin thus missing like bishops on a chess board.

Results were improved by propagating normals across a real grid rather than a discrete one. Pixel boundaries were considered real lines and messages recorded entry and exit of pixels where the line was crossed, thus intersections of normals could be deduced to sub pixel accuracy.

We suggest a scale based regional representation could be produced by incorporating information from the Curvature Primal Sketch of the SLS [1].

\section{Discussion and Conclusions}

The new algorithm's use of "voting" relates it to the Hough Transform for circle detection [2]. Figure 1 shows SLS boundary points are tangents to a circle and intersecting normals vote for the centre of this circle. The algorithm is also related to a number of shape description methods based on propagation of waves or particles from boundaries [8] and this algorithm coupled with a MIMD architecture could form a general basis for their computation.

We have shown an efficient adaptation of Brady and Scott's algorithm for a MIMD architecture is possible using the new interval decomposition method. In addition rotational symmetries can be computed simultaneously with a single analysis of the shape. Further details can be found in [9].

\section{References}

[1] H. Asada and M. Brady. The curvature primal sketch. A.I.Memo 758, MIT, February 1984.

[2] D. H. Ballard and J. Sklansky. Tumour detection in chest radiograms. Computers in Biomedical Research, 6:299-321, August 1973.

[3] M. Brady. Representing shape. In IEEE International Conference on Robotics. IEEE Computer Society Press, 1984.

[4] M. Brady and H. Asada. Smoothed local symmetries and their implementation. A.I.Memo 757, MIT, February 1984.

[5] M. Brady and G. Scott. Parallel algorithms for shape representation. In I.Page, editor, Parallel Architecture and Computer vision. Oxford university press, 1988.

[6] R. Brooks. Model-based three-dimensional interpretations of twodimensional images. IEEE Transactions on Pattern Analysis and Machine Intelligence, 5(2):140-149, March 1983.

[7] M. M. Fleck. Local rotational symmetries. In Computer Vision and Pattern Recognition, pages 332-338, Miami, Florida, 1986.

[8] S. Marshall. Review of shape coding techniques. Image and Vision Computing, 7(4):281-294, November 1989.

[9] M. W. Wright. Computation of smoothed local symmetries on a mimd architecture. Technical Report CUED/F-InfEng/Tr.68, Cambridge University Engineering Department, Cambridge, CB2 1PZ, 1991. 


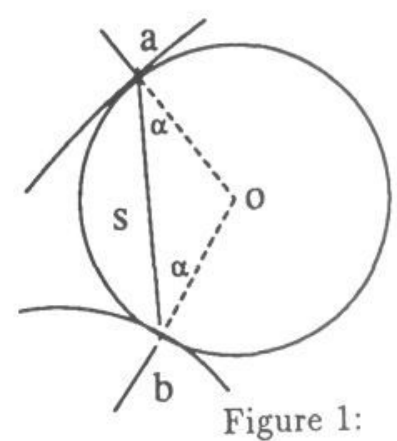

Geometry of a Local Symmetry

Rectangular shape

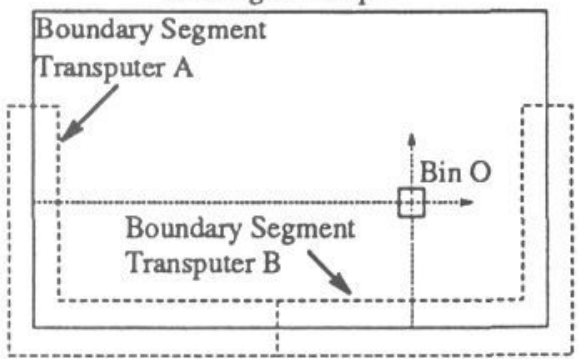

Figure 2: Propagation of Normal Vectors

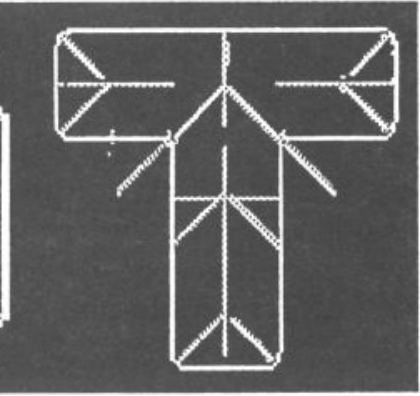

Figure 3: SLS of Square and Tee

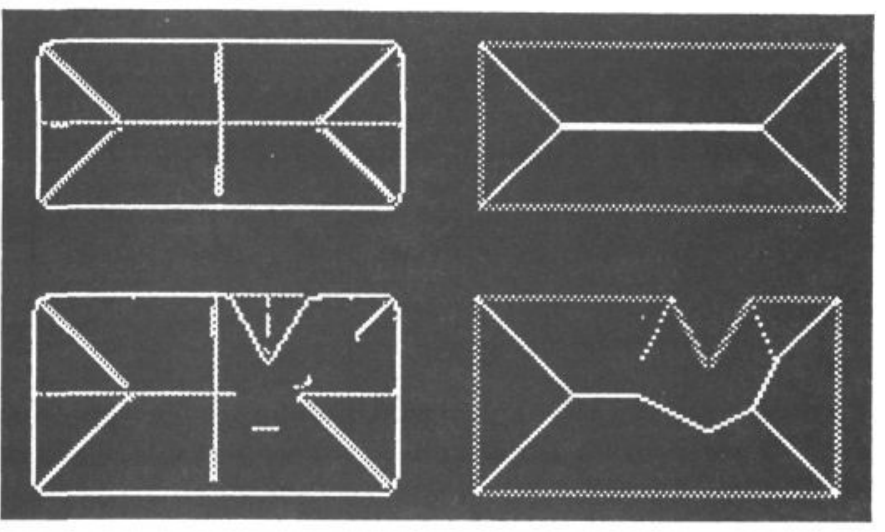

Figure 4: SLS and SAT of Rectangle and Rectangle with Notch

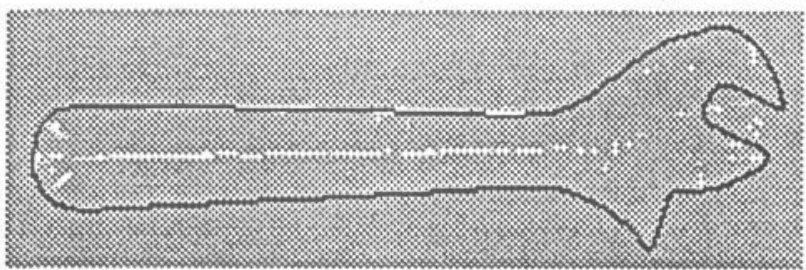

Figure 5: SLS of Wrench 